Commentary

\title{
The problem of maternal toxicity in developmental toxicity studies
}

\author{
Erminio Giavini*, Elena Menegola \\ Università degli Studi di Milano, Department of Biology, Via Celoria, 26 I-20133 Milano, Italy
}

\section{A R T I C L E I N F O}

Article history:

Available online 8 December 2011

\section{Keywords:}

Developmental toxicity studies

Maternal toxicity

\begin{abstract}
A B S T R A C T
Guidelines for developmental toxicity studies require that the highest dose(s) should induce some signs of maternal toxicity. However, the interpretation of the results is often difficult when developmentally toxic effects are recorded only at maternotoxic doses, as it is impossible to ascertain whether the developmental effects are maternally mediated or not. In order to avoid this source of misinterpretation we suggest to use in developmental toxicity tests for environmental chemicals the maximum dose unable to produce maternal toxic effects extrapolated by previous short term toxicity studies.
\end{abstract}

(c) 2011 Elsevier Inc. All rights reserved.
Guidelines for developmental toxicity studies require the administration of doses able to induce clear signs of maternal toxicity. The OECD test No. 414 (2001) indicates that "the highest dose should be chosen with the aim to induce some developmental and/or maternal toxicity (clinical signs or a decrease in body weight), but not death or severe suffering". This indication is a continuous source of problems either in the conduct of the experiments and in the interpretation of the results. The choice of a dose which is able to produce clear signs of toxicity without being lethal for some animals or inducing severe suffering (what is the limit of a severe suffering?) is rather difficult. As a consequence it is frequent to observe experiments with very severe toxic effects at the highest dose, including death or dramatic reduction of body weight. During our long term experience, serving as member of the Committee for Control and Registration of Pesticides of Italian Ministry of Public Health, we had the opportunity to examine some hundred of reports on developmental toxicity of pesticides. Although in 51/91 of acceptable reports the only reported sign of maternal toxicity was a reduction of maternal weight gain (10-20\% of the control value), in 28/91 reports maternal toxicity included sedation, loss of weight, immobility, piloerection, arched posture, chromodacryorrhea, chromorhinorrhea, ataxia, alopecia, hyperactivity, lethargy, hypersalivation, blood around the mouth, blood around vagina, diarrhea, gastric lesions. In particular in rabbits, death, sacrifice in extremis, abortion, reduced body weight were very frequent reported findings (in 58/91 reports). In some cases the frequency of maternal death and abortion was so high to preclude the evaluation of the study with the consequence of repeating the study using lower doses. Furthermore, the finding of malformations concomitant with maternal toxicity sometimes induced to perform further experiments in order to clarify the results with consequent waste of money, time, and animals. It must be stressed, according

\footnotetext{
* Corresponding author. Fax: +390250314802.

E-mail address: erminio.giavini@unimi.it (E. Giavini).
}

with our data base on pesticides, that maternal toxicity may be related to a range of effects. Severe maternotoxic effects in rat studies (reduced body weight gain $>20 \%$, clinical signs) were correlated with $39 \%$ cases of increased resorptions, $53 \%$ of reduced fetal weight, $25 \%$ of increased frequency of minor anomalies, $17 \%$ of increased frequency of malformations, and $11 \%$ of no effects. On the contrary, a moderate maternal toxicity (10-20\% reduced maternal weight gain) were correlated with $5 \%$ cases of increased resorptions, $49 \%$ of reduced fetal weight, $23 \%$ of increased frequency of minor anomalies, $37 \%$ no effects, only $2 \%$ of increased malformations. These data seem to suggest a correlation between the severity of maternal toxicity and the effects on the conceptuses, however a definite relationship cannot be extrapolated because the concurrent role of the test compound in inducing the effects must be taken into account. The role of maternal toxicity in the induction of adverse effects on conceptuses is a very long standing problem that has been faced by a number of researchers (Chahoud et al., 1999; Chernoff et al., 1989; ECETOC, 2004; Kavlock et al., 1985; Khera, 1984, 1985; Paumgartten, 2010; Rogers et al., 2005), apparently without a definite solution. First of all the definition of maternal toxicity is very poor. The guidelines are not explicative on this point, limiting to say "clinical signs or a decrease in body weight". The clinical signs indicative of maternal toxicity are numerous: from the reduced body weight gain to severe behavioral or morphological alterations. What are these symptoms expression of (pain, toxicity at the level of specific organs, altered homeostasis) is difficult to be determined. As a consequence, it is impossible to evaluate if the observed maternal symptoms have any effect on the conceptus. Moreover, in the case of developmental toxicity at maternotoxic dose levels it is impossible to discriminate between direct and maternotoxicity mediated effects.

The problem is a major concern if related to the decision of the European Commission (1992) to introduce a system for classification and labeling of chemicals. The chemicals classified as dangerous for development are divided into three categories. 
Allocation in Cat. 1 is made on the basis of human (epidemiological) data indicating that "there is sufficient evidence to establish a causal relationship between human exposure to the substance and subsequent developmental effect". Allocation in Cat. 2 or 3 is based on animal studies. In both cases it is stressed that the adverse effects on conceptus must be obtained "in appropriate animal studies in absence of signs of marked maternal toxicity, or at around the same dose levels as other toxic effects but which are not a secondary non-specific consequence of the other toxic effects". This classification has a strong impact on the chemical marketing because the chemicals that are classified must carry risk phrases such as "possible risk of harm to the unborn child" that may have serious consequences on their marketing. Furthermore, in some countries (e.g. in Brazil) registration of any pesticide that was found to be teratogenic is forbidden (Paumgartten, 2010). The difference between Cat. 2 and 3 is that the results must provide strong evidence of adverse developmental effects (Cat. 2) or some evidence of adverse developmental effects but insufficient to place the chemicals in Cat. 2. It is quite evident that this classification system is a source of continuous conflicts between regulatory agencies and manufacturers. The regulatory agencies, in order to safeguard the human health, tend to classify in Cat. 2 or 3 all chemicals able to produce signs of adverse developmental effects even in presence of maternal toxicity considering the adverse developmental effects as an intrinsic property of the test compound, not mediated by maternal toxicity. On the contrary the manufacturer position is that some adverse developmental effects (reduced fetal weight, increase of minor anomalies or variations, increase of resorptions) could be mediated by maternal toxicity and not a direct effect of the test compound on the conceptus.

During the ECETOC Workshop on maternal toxicity (2004) it has been proposed, in order to better define the maternal effects, to increase the information about maternal parameters (e.g. acute phase proteins in serum, serum $\mathrm{Zn}$ concentrations, haematology, clinical chemistry, organ weight, and histopathology). The collection of these additional information would significantly increase the cost of the studies without definitely solving the problem and producing, on the contrary, some additional questions, for example, at what time of gestation to collect these data: during the middle of organogenetic period or at termination of treatment? As the more recent guidelines (OECD, 2001) for industrial or agricultural chemicals suggest to continue the treatment "to the day prior to scheduled caesarian section", the maternotoxic manifestation observed at the end of treatment may be different from those observed at midgestation, the period of highest susceptibility for embryotoxic effects. The paper by Beyer et al. (2010) summarizes the discussions of workshops on maternal toxicity held during the Society of Toxicology, Teratology Society, and European Teratology Society meetings in 2009. The majority of the presentation stressed the reduced maternal weight gain or reduced maternal weight as main signs of maternal toxicity and correlated this signs of toxicity with reduced food intake with possible indirect consequences on fetal weight or other developmental effects. Like in several other papers/workshops a consensus was not obtained about the relationship between maternal and fetal effects. Some participants agreed that maternal toxicity can provide an explanation for some developmental effects. In this case the regulatory authorities would not label test compounds as developmental toxicants. But this assertion must be accepted on the basis of supporting data, e.g. mechanistic data provided by the manufacturers on the basis of ad hoc studies. That means a lot of time and a lot of animals to be used, without a certainty of success. On the other hand, some regulators did not agree with this position, arguing that embryofetal findings are important regardless of any relationship to maternal toxicity, in particular when the NOEL of the test article is decreased based on developmental toxicity data.
The use of a dose level with the specific aim to induce maternal toxicity, i.e. some degree of animal suffering, is also against the ethical concepts and the Directive 2010/63/EU of the European Parliament on the protection of animals used for scientific purposes which at the art. 13 (Choice of methods), §2 says "choose between procedures those which meet the following requirements: c) cause the least pain, suffering, distress or lasting harm". In our opinion the use of maternally toxic doses is just confounding without a significant improvement of the method. If the use of maternotoxic doses may make sense in testing pharmaceuticals, as the distance between the therapeutic and toxic doses can be short, in the case of environmental chemicals the exposure of pregnant women, in normal conditions excluding disasters, is at maximum the ADI that in general is a very small dose, fixed at a hundredth or less of the NOEL from long term or developmental toxicity studies. As reported in Table 1, the ratio between the highest dose used in developmental toxicity studies and the ADI is among 1000-500,000. As a consequence the use of excessively high doses in order to obtain maternal toxicity is not necessary in relation to the extrapolation to human exposure. On this purpose, the results of the EU coordinated programme on pesticide residues in food available to European consumers are relevant. A total of 11,610 samples of different vegetables and fruits from 27 member states were analyzed for 78 pesticides. In $62.1 \%$ of samples no residues were detected; in $35.7 \%$ of samples the residues were below the MRL (Maximum Residue Levels), and only $2.2 \%$ of samples exceeded the MRL (EFSA, 2008). This is a reassuring result. Although the MRL for agricultural pesticides is derived from trials performed according to good agricultural practice rather than the healthbased exposure limit of ADI, a strict correlation does exist between MRL and ADI because a proposed MRL would not be approved unless the estimated intakes based on that MRL were consistent with exposure below the ADI (Renwick, 2002). Also the workers may be protected without using maternotoxic doses. The AOEL (Admissible Operator Exposure Level) is defined as the maximum amount of active substance to which the operator may be exposed without any adverse health effects and is obtained from toxicological database in a manner similar to ADI. In some cases the compulsory use of particular personal protective equipment is also requested. According to the current EU legislation, the AOEL set for operators and workers should be also applicable to bystanders (persons in vicinity of a pesticide application).

Two recent papers, signed also by scientists working in US regulatory agencies, agree with the hypothesis of avoiding maternotoxic doses and suggest new directions in study designs and testing strategies: "for industrial and agricultural compounds found in the environment, typical human exposures tend to occur at much lower

Table 1

Ratio between the highest dose used in some developmental toxicity studies on rat to test pesticides and the admissible daily intake.

\begin{tabular}{lclr}
\hline Pesticide & Highest dose in rat $\mathrm{mg} / \mathrm{kgbw}$ & ADI $\mathrm{mg} / \mathrm{kgbw}$ & \multicolumn{1}{c}{ Ratio } \\
\hline Triadimefon & 100 & 0.01 & 10,000 \\
Tebuconazole & 120 & 0.03 & 4000 \\
Cyromazine & 600 & 0.02 & 30,000 \\
Teflubenzuron & 250 & 0.01 & 25,000 \\
Penconazole & 300 & 0.03 & 10,000 \\
Endosulfan & 6 & 0.006 & 1000 \\
Diazinon & 100 & 0.0002 & 500,000 \\
Imazalil & 40 & 0.01 & 4000 \\
Metiram & 160 & 0.03 & 5300 \\
Myclobutanil & 460 & 0.03 & 15,300 \\
Amitraz & 12 & 0.003 & 4000 \\
Clofentezine & 3200 & 0.02 & 160,000 \\
Buprofezin & 800 & 0.01 & 80,000 \\
Cycloxydim & 800 & 0.07 & 11,400 \\
\hline
\end{tabular}


levels, often orders of magnitude lower, than the doses used in test animals. Omissis. Nonetheless, most testing guidelines for chemicals still specify gavage administration of maximally tolerated dose levels as the default, even in cases where human exposure are known to be orders of magnitude lower. Omissis. The negative consequences of this practice include unnecessary animal use, cost, and effort as the irrelevant findings generated are further investigated. This testing scheme also can lead to the erroneous classification and labeling of compounds which pose little risk to humans" (Carney et al., 2011). The second paper (Brannen et al., 2011) is a summary of the discussion of a workshop on developmental toxicology - new directions, held by ILSI and HESI. In the chapter "Dose selection" is reported: "there were comments throughout the discussion about the need to move away from high, maternally toxic doses that may be meaningless for human exposure. For industrial chemicals, an alternative approach gaining some traction is the setting of the high dose level based on toxicokinetics, specifically to avoid dose levels which saturate metabolic and excretory processes and result in non linear kinetics. Omissis. It was the consensus of the majority of attendees that a more rational upper limit should be adopted".

Actually, another relevant point should be addressed: the dosedependent transitions. A transition is a change with increasing dose in key underlying kinetic and/or dynamic factors that influence the mechanism responsible for the observed toxicity, resulting in a change in the relationship of the response rate as function of dose (Slikker et al., 2004a). The saturable or inducible factors such as absorption, distribution, elimination, metabolism, besides receptor interactions and altered homeostasis are the basis for dose-dependent transitions. When very high doses are used in toxicity tests, it is likely to be in the range of dose-dependent transitions, obtaining data useless for risk assessment. Just one example related to developmental toxicity reported by Slikker et al. (2004b): high doses of ethylene glycol (EG, $>1000 \mathrm{mg} / \mathrm{kg}$ ) resulted in congenital malformations in rodents. Further in vivo and in vitro studies established that the congenital malformations were due to a metabolite of EG, glyoxylic acid (GA). The pharmacokinetics of EG showed the primary importance of a dose-dependent transition involving saturation of GA oxidation leading to accumulation of this toxic metabolite at high dose levels.

In conclusion, the use of maternotoxic doses in developmental toxicity studies of environmental chemical is not to be recommended for different reasons: (1) may produce results difficult to be interpreted and source of continuous discussions between sponsors and regulatory agency often resulting in additional but not conclusive experiments; (2) may lead to a misclassification of chemicals; (3) the doses are thousand times the ADIs; (4) the obtained results may be misleading because of dose-dependent transitions.

On the basis of what exposed above, the only way to bypass the problem of maternal toxicity and its pernicious influence on the interpretation of developmental toxicity studies, is to avoid the use of maternotoxic doses in such kind of studies when performed on environmental chemicals. In order to avoid this source of misinterpretation we suggest that the currently used maximum tolerated dose be replaced with new, more refined criteria for dose level selection. The highest dose should be a dose sufficiently high but unable to induce overt clinical signs of toxicity or reduction of maternal body weight gain. We suggest that the highest dose may be chosen on the basis of previous short term toxicity studies and on the basis of a range finding developmental toxicity study performed in a range of doses, for example, between the mid and the highest tested doses in a 28-day general toxicity study. If the test compound has an embryotoxic potency, this dose will produce more clear developmental effects without interferences of maternal toxicity avoiding the difficulties of data interpretation when the developmental effects are observed at maternally toxic dose levels and will be in any case sufficiently higher than the ADI for the safety of the human conceptuses.

\section{Conflict of interest statement}

The authors declare that there are no conflicts of interest.

\section{References}

Beyer, B.C., Chernoff, N., Danielsson, B.R., et al., 2010. ILSI/HESI maternal toxicity workshop summary: maternal toxicity and its impact on study design and data interpretation. Birth Defects Res. B 92, 36-51.

Brannen, K.C., Fenton, S.E., Hansen, D.K., Harrouk, W., Kim, J.H., Shuey, D., 2011. Developmental toxicology - New directions workshop: refining testing strategies and study designs. Birth Defects Res. B 92, 404-412.

Carney, W.E., Ellis, A.L., Tyl, R.W., Foster, P.M.D., Scialli, A.R., Thompson, K., Kim, J., 2011. Birth Defects Res. B 92, 395-403.

Chahoud, I., Ligensa, A., Dietzel, L., Faqi, A.S., 1999. Correlation between maternal toxicity and embryo/fetal effects. Reprod. Toxicol. 13, 375-381.

Chernoff, N., Rogers, J.M., Kavlock, R.J., 1989. An overview of maternal toxicity and prenatal development: considerations for developmental toxicity hazard assessments. Toxicology 59, 111-125.

ECETOC, 2004. Influence of maternal toxicity in studies on developmental toxicity. Workshop. March 2nd ECETOC Workshop report N.4. Brussels, Belgium.

European Food Safety Authority, 2010. 2008 annual report on pesticide residues according to article 32 of regulation (EC) No 396/2005. EFSA Journal, 8: 16462088.

European Commission, 1992. Council Directive of 5th June 1992 amending for the 7th time Directive 67/548/EEC. Off. J. Eur. Commun. L.154/1.

Kavlock, R.J., Chernoff, N., Rogers, E.H., 1985. The effect of acute maternal toxicity on fetal development in the mouse. Teratogen. Carcinog. Mutag. 5, 3-13.

Khera, K.S., 1984. Maternal toxicity - A possible factor in fetal malformations in mice. Teratology 29, 411-416.

Khera, K.S., 1985. Maternal toxicity: a possible etiologic factor in embryo-fetal deaths and fetal malformations of rodent-rabbit species. Teratology 31, 129153.

OECD, 2001. Guideline for the testing of chemicals, 414. Prenatal developmental toxicity study. Organization for Economic Cooperation and Development, Paris, France.

Paumgartten, F.J.R., 2010. Influence of maternal toxicity on the outcome of developmental toxicity studies. J. Toxicol. Environ. Health A 73, 944-951.

Renwick, A.G., 2002. Pesticide residue analysis and its relationship to hazard characterization (ADI/ARfD) and intake estimations (NEDI/NESTI). Pest Manag. Sci. 58, 1073-1082.

Rogers, J.M., Chernoff, N., Keen, C.L., Daston, G.P., 2005. Evaluation and interpretation of maternal toxicity in segment II studies: issues, some answers, and data need. Toxicol. Appl. Pharmacol. 207, S367-S374.

Slikker, Wjr., Andersen, M.E., Bogdanffy, M.S., 2004a. Dose-dependent transitions in mechanisms of toxicity. Toxicol. Appl. Pharmacol. 201, 203-225.

Slikker, Wjr., Andersen, M.E., Bogdanffy, M.S., 2004b. Dose-dependent transitions in mechanisms of toxicity: case studies. Toxicol. Appl. Pharmacol. 201, 226-294. 